\title{
Untangling neuroinflammation in amyotrophic lateral sclerosis
}

\section{$\underline{\text { Strapline }}$}

Chitinase levels are linked to rate of progression and provide a new foothold on optimizing therapeutic targeting of neuroinflammatory pathways.

\section{Main text}

A broad body of evidence supports both pathogenic and neuroprotective roles for inflammation in $\mathrm{ALS}^{1}$ but trials of several immunomodulatory drugs have not yielded a beneficial effect in an increasingly complex disorder with multiple upstream cellular causes ${ }^{2}$. There has been interest in proteins involved in the microglial response as potential biomarkers for well over a decade ${ }^{34}$. Recently this has focused on a group of three chitinase proteins, thought to be macrophage-derived, identified in proteomic analysis of cerebrospinal fluid (CSF) taken from ALS patients ${ }^{56}$. Chitinases have also been studied the pathologically-related disorder frontotemporal dementia (FTD ${ }^{78}$. Although chitin is not produced by mammals, it is suggested that chitinases might act upon $\mathrm{N}$-acetylglucosaminecontaining extracellular matrix polymers, such as hyaluronan or keratan sulphate, present in the mammalian central nervous system. Chitotriosidase 1 (CHIT1) is an active chitinase, whilst YKL-40 (Chitinase-3-like protein 1, CH3L1) and YKL-39 (Chitinase-3-like protein 2, $\mathrm{CHI} \mathrm{L} 2$ ) are inactive chitinases involved in innate immunity.

In this issue, Gille, De Schaepdryver et al measured CSF levels of CHIT1, YKL-40 and macrophage chemoattractant protein-1 (MCP-1) in 105 ALS patients, 102 patients with neurological diseases distinct from ALS, and a group of 16 patients initially suspected to have ALS in whom the diagnosis was subsequently excluded (termed ALS mimics). The finding of elevated levels of the three proteins in ALS patients compared with other groups is in keeping with the published literature ${ }^{3-69-11}$. The unimpressive classifier performance of CHIT1 and YKL-40 in distinguishing ALS from mimics is similar to previous studies ${ }^{6}{ }^{10} ; \mathrm{MCP}-1$ also performed poorly as an independent classifier. CHIT1 and YKL-40 levels were linked to rate of disability progression, though a previously-identified weak correlation of MCP-1 with rate of progression was not observed ${ }^{3}$. The study also demonstrated survival associations with both MCP-1 and YKL-40 in multivariate models, but not CHIT1, in contrast to a previous study which reported an association only with $\mathrm{CHIT} 1^{10}$. Levels of all proteins correlated inversely with survival from in univariate analysis. The authors also noted higher CHIT1 levels in those with greater regional burden of disease, so that there appears to be consistency across studies for CHIT1 levels as a marker of absolute disease progression. The present study did not screen for the presence of a CHIT1 polymorphism which can lead to reduced CHIT1 activity ${ }^{12}$, though a previous study reported that this did not appear to influence the severity of ALS ${ }^{7}$. As noted in previous studies, serum levels of CHIT1 and YKL40 were not significantly elevated.

CSF CHIT1 levels have been linked to the degree of microglial activity within the spinal cord ${ }^{9}$. YKL-40 levels have been correlated with the burden of cognitive dysfunction and extent of clinical upper motor neuron involvement in $\mathrm{ALS}^{10}$. In a study comparing FTD patients and controls there was marked elevation of CSF YKL-40 and the astrocytic protein glial fibrillary 
acidic protein, but not $\mathrm{CHIT}^{7}{ }^{7}$. Pathological data in Alzheimer's disease suggest that YKL-40 may be predominantly astrocytic rather than microglial in origin ${ }^{13}$. Taken together, these findings attest to the multi-dimensional nature of the neuroinflammatory component of neurodegeneration in ALS, in which CSF CHIT1 levels reflect microglial activity within the spinal cord and YKL-40 levels represent astroglial activity within the brain.

Immunomodulatory therapeutic approaches must necessarily be highly nuanced. Measuring the CSF inflammatory profile in ALS may have unrealised value in the stratification and appropriate selection of participants for future trials, leading the way in an era of increasingly personalised therapy.

\section{References}

1. Evans MC, Couch $\mathrm{Y}$, Sibson N, et al. Inflammation and neurovascular changes in amyotrophic lateral sclerosis. Molecular and cellular neurosciences 2013;53:34-41. doi: 10.1016/j.mcn.2012.10.008 [published Online First: 2012/11/01]

2. Talbot K, Feneberg E, Scaber J, et al. Amyotrophic lateral sclerosis: the complex path to precision medicine. J Neurol 2018;265(10):2454-62. doi: 10.1007/s00415-018-8983-8

3. Tanaka M, Kikuchi $H$, Ishizu $T$, et al. Intrathecal upregulation of granulocyte colony stimulating factor and its neuroprotective actions on motor neurons in amyotrophic lateral sclerosis. J Neuropathol Exp Neurol 2006;65(8):816-25. doi: 10.1097/01.jnen.0000232025.84238.e1

4. Wilms H, Sievers J, Dengler R, et al. Intrathecal synthesis of monocyte chemoattractant protein-1 (MCP-1) in amyotrophic lateral sclerosis: further evidence for microglial activation in neurodegeneration. J Neuroimmunol 2003;144(1-2):139-42.

5. Varghese AM, Sharma A, Mishra P, et al. Chitotriosidase - a putative biomarker for sporadic amyotrophic lateral sclerosis. Clinical proteomics 2013;10(1):19. doi: 10.1186/1559-0275-10-19 [published Online First: 2013/12/04]

6. Thompson AG, Gray E, Thezenas ML, et al. Cerebrospinal fluid macrophage biomarkers in amyotrophic lateral sclerosis. Annals of neurology 2018;83(2):258-68. doi: 10.1002/ana.25143 [published Online First: 2018/01/14]

7. Oeckl $P$, Weydt $P$, Steinacker $P$, et al. Different neuroinflammatory profile in amyotrophic lateral sclerosis and frontotemporal dementia is linked to the clinical phase. Journal of neurology, neurosurgery, and psychiatry 2019;90(1):4-10. doi: 10.1136/jnnp-2018318868 [published Online First: 2018/09/19]

8. Vucic S. Differences in inflammatory profiles between ALS and FTD. J Neurol Neurosurg Psychiatry 2019;90(1):1. doi: 10.1136/jnnp-2018-319377

9. Steinacker P, Verde F, Fang L, et al. Chitotriosidase (CHIT1) is increased in microglia and macrophages in spinal cord of amyotrophic lateral sclerosis and cerebrospinal fluid levels correlate with disease severity and progression. J Neurol Neurosurg Psychiatry 2018;89(3):239-47. doi: 10.1136/jnnp-2017-317138

10. Thompson AG, Gray E, Bampton A, et al. CSF chitinase proteins in amyotrophic lateral sclerosis. J Neurol Neurosurg Psychiatry 2019 doi: 10.1136/jnnp-2019-320442

11. Mitchell RM, Freeman WM, Randazzo WT, et al. A CSF biomarker panel for identification of patients with amyotrophic lateral sclerosis. Neurology 2009;72(1):14-9. doi: 10.1212/01.wnl.0000333251.36681.a5 
12. Lee $P$, Waalen J, Crain K, et al. Human chitotriosidase polymorphisms G354R and A442V associated with reduced enzyme activity. Blood Cells Mol Dis 2007;39(3):353-60. doi: 10.1016/j.bcmd.2007.06.013

13. Querol-Vilaseca M, Colom-Cadena M, Pegueroles J, et al. YKL-40 (Chitinase 3-like I) is expressed in a subset of astrocytes in Alzheimer's disease and other tauopathies. $J$ Neuroinflammation 2017;14(1):118. doi: 10.1186/s12974-017-0893-7 